Revista Brasil. Bot., V.25, n.1, p.35-42, mar. 2002

\title{
Ruellia brevifolia (Pohl) Ezcurra (Acanthaceae): fenologia da floração, biologia da polinização e reprodução
}

\author{
MARIA R. SIGRIST ${ }^{1,3}$ e MARLIES SAZIMA ${ }^{2}$
}

(recebido: 21 de fevereiro de 2001; aceito: 14 de novembro de 2001)

\begin{abstract}
Ruellia brevifolia (Pohl) Ezcurra (Acanthaceae): flowering phenology, pollination biology and reproduction). The reproductive biology of Ruellia brevifolia was studied in two populations of southeastern Brazil. R. brevifolia is a shrublet that grows in dense clumps in open but shaded areas. This Acanthaceae blooms all year round and produces chasmogamous $(\mathrm{CH})$ and cleistogamous (CL) flowers during the rainy and the dry season, respectively. Cleistogamy is of the "clymatic" type, and produces seeds in the most stressful period of the year. CH flowers are tubular, red and odourless, protogynous, and may be herkogamous or not; anthesis is diurnal, and they last two days. Nectar is produced in small amounts $(5.2 \mu \mathrm{l} \pm 2.36)$ and its concentration is about 30\%. CL flowers resemble CH buds, except for their pale color. Pollen/ovule ratio of CL flowers is $60 \%$ lesser than that of $\mathrm{CH}$ flowers because of the small amount of pollen grains. This Ruellia is self-compatible, but protogyny and herkogamy favour allogamy; spontaneous self-pollination in $\mathrm{CH}$ flowers seems to be related to the non herkogamous flowers. The hummingbird Amazilia lactea and the butterflies Heliconius etilla narcaea and Phoebis argante are the major pollinators of $R$. brevifolia. The hummingbirds visit $R$. brevifolia only during flowering peaks, when there is a high number of flowers. It seems plausible that both the small nectar volume per flower and the trap-line foraging strategy of A. lactea promote mainly outcrossing. The butterflies visit flowers mainly in the same plant group, but during low floral density periods they fly greater distances also favouring outcrossing.
\end{abstract}

RESUMO - (Ruellia brevifolia (Pohl) Ezcurra (Acanthaceae): fenologia da floração, biologia da polinização e reprodução). A biologia reprodutiva de Ruellia brevifolia foi estudada em populações naturais do sudeste brasileiro. R. brevifolia é um subarbusto que forma agrupamentos densos em áreas abertas mas sombreadas e floresce o ano todo, produzindo flores casmógamas $(\mathrm{CH})$ e cleistógamas (CL) nas estações úmida e seca, respectivamente. A cleistogamia é do tipo climática, originando sementes no período de maior estresse ambiental. Flores $\mathrm{CH}$ são inodoras e possuem corola tubulosa e vermelha, sendo hercogâmicas ou não; apresentam protoginia, antese diurna e duram dois dias. Néctar é o principal recurso floral, sendo produzido em baixa quantidade $(5,2 \mu \mathrm{l} \pm 2,36)$ e com concentração de açúcares em torno de $30 \%$. Flores $\mathrm{CL}$ assemelham-se a botões jovens de flores $\mathrm{CH}$ e apresentam redução de tamanho principalmente no androceu e na corola. A razão pólen/óvulo das flores CL é ca. de 60\% menor que a das flores $\mathrm{CH}$ devido a menor quantidade de grãos de pólen. $R$. brevifolia é autocompatível embora alogamia seja favorecida pela protoginia e pelas flores hercogâmicas. Autopolinização espontânea parece estar relacionada às flores $\mathrm{CH}$ não hercogâmicas. Beija-flores, principalmente Amazilia lactea, e borboletas, em especial Heliconius etilla narcaea e Phoebis argante, são os polinizadores de $R$. brevifolia. Os beija-flores visitam $R$. brevifolia apenas durante os picos de floração, quando há elevado número de flores. A baixa quantidade de néctar por flor, bem como as visitas de A. lactea em "linha-de-captura", provavelmente, promovem a polinização cruzada. Embora as borboletas visitem especialmente dentro e entre agrupamentos próximos, também visitam as flores nos períodos de baixa densidade floral, quando se deslocam a maiores distâncias, favorecendo a polinização cruzada.

Key words - Floral biology, herkogamy, cleistogamy, ornithophily, psicophily

\section{Introdução}

Na América do Sul meridional o gênero Ruellia consta ca. de 23 espécies que apresentam grande diversidade na morfologia floral, fato que é associado a um amplo espectro de polinizadores (Ezcurra 1993).

\footnotetext{
1. Universidade Federal do Mato Grosso do Sul, Departamento de Biologia, Centro de Ciências Biológicas e da Saúde, Caixa Postal 549, 79070-900 Campo Grande, MS, Brasil.

2. Universidade Estadual de Campinas, Instituto de Biologia, Departamento de Botânica, Caixa Postal 6109, 13083-970 Campinas, SP, Brasil.

3. Autor para correspondência: msigrist@nin.ufms.br
}

As relações entre as flores de espécies de Ruellia e seus prováveis polinizadores têm sido inferidas, principalmente, a partir de características morfológicas (Knuth 1905, Long 1971, Ramamoorthy 1988, Ezcurra \& Azkue 1989), atribuindo-lhes síndromes de melitofila, psicofilia, ornitofilia e quiropterofilia. Entre estas síndromes, ornitofilia foi constatada em Ruellia asperula, na caatinga do NE brasileiro (Machado \& Sazima 1995). Em plantas cultivadas de $R$. brevifolia Piovano et al. (1995) registraram visitas de Colibri sp., embora não mencionem se esta espécie de beija-flor é o polinizador. 
No gênero Ruellia, autocompatibilidade parece ser freqüente (Machado \& Sazima 1995, Piovano et al. 1995) e, em várias espécies deste gênero, cleistogamia é bastante comum, característica que parece estar associada a fatores ambientais como período de seca e/ ou altas-baixas temperaturas (Long 1977, Sell 1977, Lord 1981, Raghuvanshi et al. 1981).

Ruellia brevifolia tem ampla distribuição nas regiões tropicais da América do Sul austral, sendo cultivada como planta ornamental em diversas regiões quentes do mundo (Ezcurra 1989). Esta espécie é comum em sub-bosques de áreas abertas de mata sujeitas a alterações, tais como clareiras e beiras de trilhas, sendo também encontrada com freqüência em áreas sombreadas (Ezcurra 1989).

Neste estudo apresentamos a fenologia da floração, a biologia da polinização e o sistema de reprodução em populações naturais de $R$. brevifolia do sudeste brasileiro. Discutimos estes aspectos em relação aos visitantes florais, bem como a ocorrência de cleistogamia face a fatores ambientais.

\section{Material e métodos}

As plantas de R. brevifolia estudadas ocorrem agrupadas em locais sombreados de borda de mata semidecídua (Reserva Municipal de Santa Genebra = RSG) e em área sombreada da

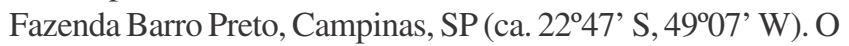
clima da região é caracterizado por uma estação seca e fria de maio a agosto, uma estação úmida e quente de novembro a fevereiro e duas estações transicionais, março-abril e setembro-outubro, caracterizadas por variações na precipitação e temperatura (Morellato \& Leitão Filho 1996). O trabalho de campo estendeu-se de abril/1988 a junho/1989, com observações adicionais em fevereiro-agosto/1990 e março-junho/1991.

Foram anotadas informações sobre a distribuição e o hábito das plantas, bem como a ocorrência de estruturas de reprodução vegetativa. Na RSG, a floração da espécie foi acompanhada mensalmente, a cada 15-25 dias, quando ocorrem os picos de floração, sendo verificada a presença (alta ou baixa) de flores casmógamas (CH) e/ou cleistógamas (CL), em 5 a 14 agrupamentos, bem como a duração dos picos de floração. Na caracterização da intensidade desta fenofase foram utilizados os termos alta, quando a maioria das plantas ( $\geq 50 \%$ ) dos agrupamentos observados apresentava botões/ flores e baixa, quando poucas plantas estavam floridas.

Dados sobre a morfologia e coloração dos dois tipos florais ( $\mathrm{CH}$ e $\mathrm{CL}$ ), odor e néctar foram registrados, bem como início e duração da antese de flores $\mathrm{CH}(\mathrm{n}=23)$, acompanhadas desde a pré-antese. Na morfometria floral foram utilizadas 20 flores de cada tipo ( $\mathrm{CH} \mathrm{e} \mathrm{CL}$ ); adicionalmente foram coletadas 110 flores $\mathrm{CH}$, em 21 plantas de 8 agrupamentos, para medir o comprimento do estilete/estigma em relação aos estames/ anteras.

O volume de néctar acumulado até o final da manhã e a concentração de açúcares no néctar foram medidos com microseringa e refratômetro de bolso, respectivamente, a partir de 15 flores $\mathrm{CH}$ de primeiro dia, ensacadas na pré-antese.

A viabilidade do pólen foi determinada com carmin acético (Dafni 1992) e/ou verde malaquita/fucsina ácida (Alexander 1980), sendo os grãos de pólen considerados viáveis quando totalmente corados e esféricos. O percentual de viabilidade foi calculado para 32 flores $\mathrm{CH}$ ( 27 de primeiro dia e 5 de segundo dia) e 9 flores $C L$, provenientes de plantas de 3 agrupamentos. Foram contados pelo menos 100 grãos por flor.

A razão P/O (Cruden 1977) foi determinada utilizando-se 10 botões de cada tipo floral, coletados em plantas de dois agrupamentos. Os grãos de pólen de quatro anteras em flores $\mathrm{CL}$ e de uma antera em flores $\mathrm{CH}$ foram quantificados com contador manual, adotando método semelhante ao de Piovano et al. (1995). Nos dois tipos florais, o número de óvulos foi contado após dissecação do ovário sob estereomicroscópio. O Teste T (Sokal \& Rohlf 1979) foi utilizado para comparação da razão $\mathrm{P} / \mathrm{O}$. A receptividade do estigma foi verificada com peróxido de hidrogênio (Dafni 1992) e Sudam III glicerinado (Johansen 1940).

Nos estudos sobre o sistema reprodutivo foram feitos experimentos de autopolinização espontânea e manual, polinização cruzada e para verificar agamospermia (cf. Radford et al. 1974) em flores $\mathrm{CH}$ de primeiro dia previamente ensacadas, utilizando-se plantas de pelo menos quatro agrupamentos. Flores $\mathrm{CH}$ e CL foram marcadas para verificar o desenvolvimento de frutos em condições naturais. Para verificar a viabilidade das sementes resultantes dos tratamentos de autopolinização manual $(n=14)$ e espontânea $(n=12)$, polinização cruzada $(n=6)$, geitonogamia e condições naturais $(n=4)$, algumas sementes coletadas foram escolhidas ao acaso e colocadas para germinar em placas de petri revestidas com papel de filtro úmido, sob condições de luminosidade e temperatura $\left(30^{\circ} \mathrm{C}\right)$ constantes, em câmara climática. As sementes foram consideradas viáveis após emitirem radículas. O índice de autopolinização espontânea (IAS), o índice de autoincompatibilidade (ISI) e a eficácia reprodutiva (cf. Sobrevila \& Arroyo 1982) foram calculados (tabela 1).

O comportamento dos visitantes florais foi estudado através de observação visual direta e fotografias. Foram registrados o horário e a frequiência das visitas, bem como o recurso procurado e o modo de explorar as flores (Machado \& Sazima 1995). As observações foram realizadas principalmente no período da manhã, entre 7:00-13:00 h, às vezes até 18:00 h, durante 21 dias, totalizando 63 horas de observações.

Material testemunho de $R$. brevifolia está depositado no herbário da Universidade Estadual de Campinas (UEC 064307). 
Tabela 1. Resultados dos experimentos sobre o sistema reprodutivo de Ruellia brevifolia. IAS= índice de autopolinização espontânea (\% frutos formados por flores autopolinizadas espontaneamente/\% flores autopolinizadas manualmente); ISI = índice de autoincompatibilidade (\% frutos formados por flores autopolinizadas manualmente/\% flores submetidas à polinização cruzada); Eficácia reprodutiva (\% frutos formados em condições naturais/\% frutos formados por polinização cruzada) (sensu Sobrevila \& Arroyo 1982).

\begin{tabular}{|c|c|c|}
\hline \multirow[t]{3}{*}{ Testes } & \multicolumn{2}{|c|}{ Tipo floral } \\
\hline & $\mathrm{CH}$ & $\mathrm{CL}$ \\
\hline & $\%$ (n. frutos/ n. flores) & $\%$ (n. frutos/ n. flores) \\
\hline Autopolinização espontânea & $30(11 / 36)$ & - \\
\hline Autopolinização manual & $95(21 / 22)$ & - \\
\hline Geitonogamia & $100(12 / 12)$ & - \\
\hline Polinização cruzada & $90(28 / 31)$ & - \\
\hline Emasculação & $0(0 / 23)$ & - \\
\hline Condições naturais & $56(23 / 41)$ & $33(6 / 18)$ \\
\hline IAS & 0,32 & - \\
\hline ISI & 1,06 & - \\
\hline Eficácia reprodutiva & 0,62 & - \\
\hline
\end{tabular}

\section{Resultados}

Nos locais de estudo $R$. brevifolia forma agrupamentos densos, descontínuos, em ambientes sujeitos a alterações, ocupando áreas de 10 a $80 \mathrm{~m}^{2}$. É um subarbusto perene com até 2,0 m de altura. As inflorescências são cimeiras axilares compostas por dicásios, que apresentam flores casmógamas $(\mathrm{CH})$ e/ou cleistógamas (CL), de acordo com a época do ano. As populações apresentam flores ao longo do ano, sendo que as flores $\mathrm{CH}$ ocorrem principalmente na estação úmida e as flores CL predominam durante a estação seca (figura 1). O desenvolvimento dos botões e a abertura das flores $\mathrm{CH}$ são relativamente sincrônicos intra e interagrupamentos, promovendo picos de floração nos agrupamentos que duram ca. 7-10 dias; nos intervalos entre estes picos, ca. 20-25 dias, há pouca ou nenhuma flor.

As flores $\mathrm{CH}$ são zigomorfas e tubulosas (figura 2a), ligeiramente pendentes e inodoras. A corola mede 20 a $32 \mathrm{~mm}$ de comprimento, sendo estreita na base, alargada na porção mediana, afunilando-se ligeiramente na entrada do tubo (figura 2a) e terminando em cinco pequenos lobos. Externamente a corola é vermelha (figura 3), sendo o interior amarelo claro com nervuras avermelhadas. Os estames são brancos, isodínamos, introrsos e subexsertos, com anteras rimosas. O estilete é branco e termina em estigma bífido, que pode situarse no mesmo nível (29\% - flores não hercogâmicas), à frente (42\%, figura $2 \mathrm{a})$ ou atrás das anteras (29\%), resultando em $71 \%$ de flores hercogâmicas; na mesma

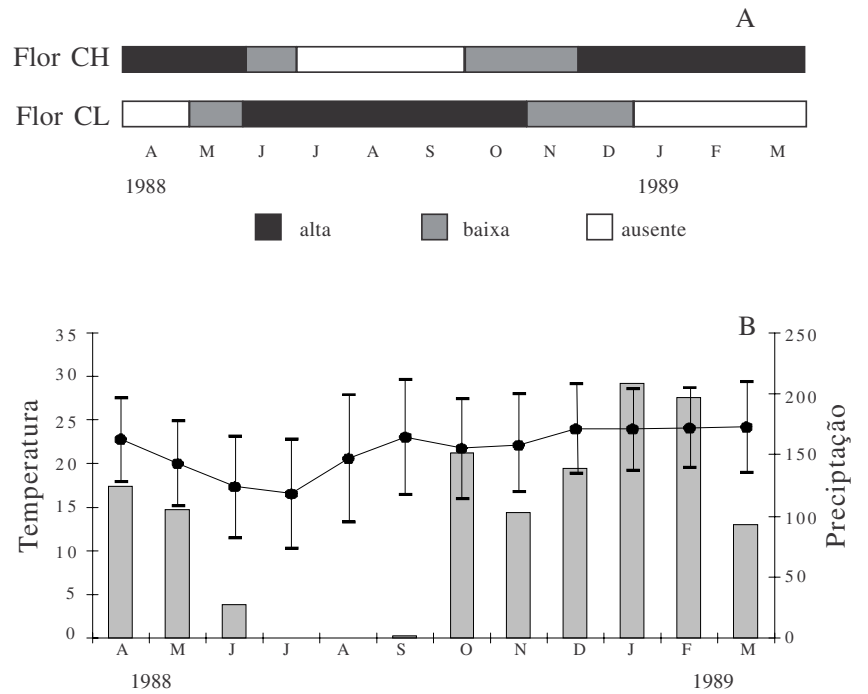

Figura 1. Fenologia da floração de Ruellia brevifolia e dados meteorológicos da região de Campinas. A. Ocorrência de flores $\mathrm{CH}$ e CL ao longo do ano (alta = a maioria das plantas dos agrupamentos apresenta flores; baixa $=$ poucas plantas apresentam flores). B. Temperatura $\left({ }^{\circ} \mathrm{C}\right)(\bullet \bullet)$ e precipitação $(\mathrm{mm})$ (barras) referentes ao período de estudo. Dados fornecidos pela Estação Meteorológica de Instituto Agronômico de Campinas.

planta pode haver flores com e sem hercogamia. $\mathrm{O}$ nectário é arredondado, situando-se abaixo do ovário (figura 2a) e produz néctar desde a fase de botão até a murcha da flor. A concentração de açúcares no néctar é de $29,35 \% \pm 6,21$ e o volume é ca. de $5,2 \mu \mathrm{l} \pm 2,36$. As flores são protogínicas e duram dois dias. Não há horário definido para a abertura das flores, embora muitos botões 

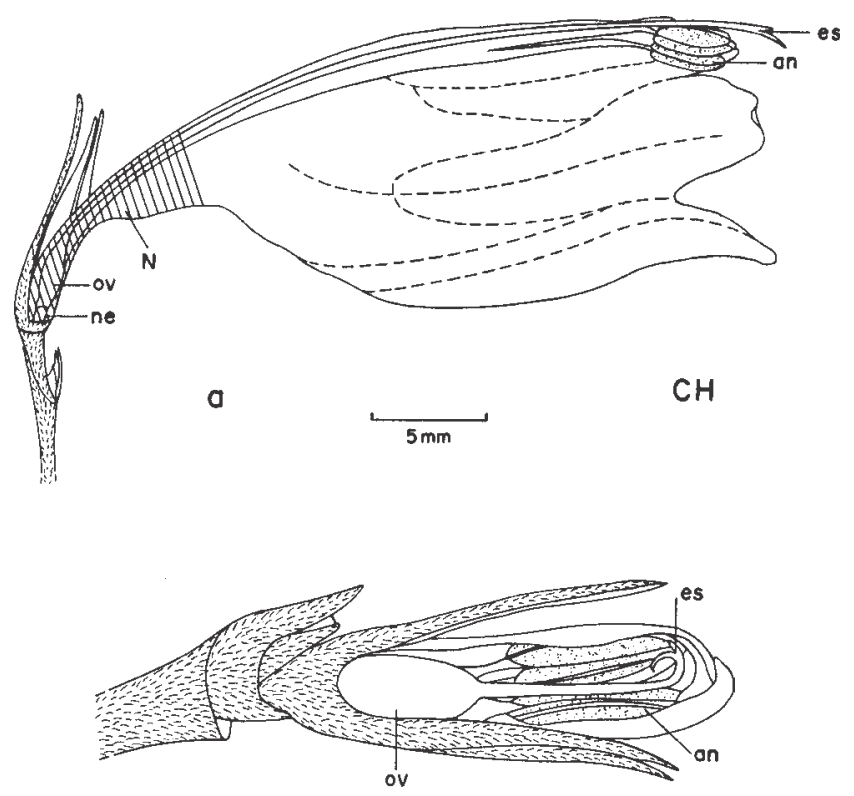

b

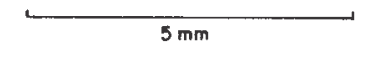

$C L$

Figura 2. Flores de Ruellia brevifolia: a) flor CH hercogâmica com néctar $(\mathrm{N})$ acumulado na base (hachureado) da corola e estigma (es) situado à frente das anteras (an); notar o nectário (ne) localizado abaixo do ovário (ov). b) flor CL com estigma em forma de gancho, situado junto às anteras.

abram, principalmente, no período da manhã. O início da antese se caracteriza pelo afastamento lento dos lobos da corola (ca. duas horas). Durante este período as anteras estão fechadas, mas o estigma está receptivo, caracterizando a fase feminina da flor. Duas a quatro horas após o início da antese as anteras estão abertas, caracterizando a fase hermafrodita. No final do segundo

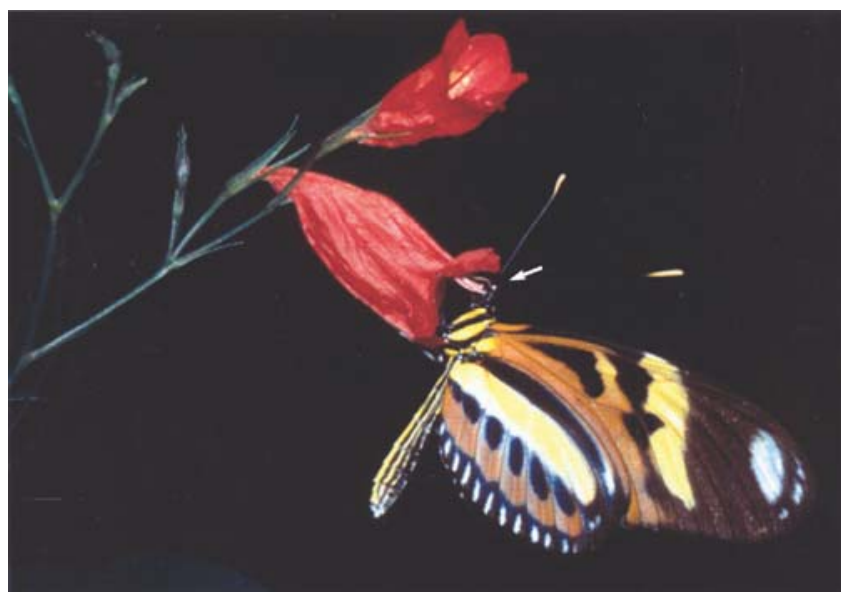

Figura 3. A borboleta Heliconius ethilla narcaea visitando flor CH de Ruellia brevifolia; notar o contato da cabeça da borboleta com os órgãos reprodutivos da flor (seta). dia as anteras geralmente estão amareladas e sem pólen e os lobos do estigma não estão mais expostos.

As flores CL (figura 2b) assemelham-se a botões jovens de flores $\mathrm{CH}$, exceto pela coloração das pétalas. Nestas flores o cálice é vináceo e possui dimensões semelhantes às da corola (5-8 mm comprimento), que é de cor rósea próximo à base e amarelada no ápice. $\mathrm{O}$ ovário e os óvulos são ligeiramente menores que o das flores $\mathrm{CH}$ e o estilete e filetes são curtos (ca. 2-3 mm comprimento). As anteras, de tamanho similar às das flores $\mathrm{CH}$, estão dispostas ao redor do estigma (figura $2 \mathrm{~b}$ ) de modo que, ao abrirem, o pólen contata a superfície estigmática (figura $2 b$ ). O estigma é bem desenvolvido e tem formato de gancho (figura $2 b$ ).

A razão P/O das flores CH é 309,1 $\pm 78,93$ e a das CL 120,7 $\pm 37,61$, respectivamente, sendo significativamente diferente $(\mathrm{P}<0,02$, teste $\mathrm{T})$. O número de óvulos é similar entre os dois tipos florais $(\mathrm{CH}=12,3 \pm 0,95 \mathrm{e}$ $\mathrm{CL}=10,9 \pm 1,29)$, mas em flores $\mathrm{CH}$ a quantidade de pólen é maior $(3798 \pm 1010,16)$ que em flores CL $(1307$ $\pm 366,37)$. As flores $\mathrm{CH}$ apresentaram $94,2 \%( \pm 3,6)$ de viabilidade polínica no primeiro dia e $89,9 \%$ ( $\pm 10,7)$ no segundo dia, enquanto nas flores CL a viabilidade do pólen foi menor, em média $81,4 \%( \pm 4,4)$.

Os resultados sobre o sistema reprodutivo de $R$. brevifolia estão resumidos na tabela 1. Esta espécie é autocompatível, ocorrendo autopolinização espontânea. Em todos os tratamentos houve produção de sementes viáveis: ca. de $65 \%$ de viabilidade nas sementes oriundas de autopolinização manual e polinização cruzada, $75 \%$ sob condições naturais e geitonogamia e $42 \%$ após autopolinização espontânea. Nesta espécie não ocorre agamospermia. Flores $\mathrm{CH}$ em condições naturais formaram mais frutos que as autopolinizadas espontaneamente e que as CL em condições naturais. Os frutos são secos, do tipo cápsulas loculicidas, que amadurecem em ca. de 30-40 dias e abrem explosivamente (ver Witztum \& Schulgasser 1995). A reprodução vegetativa é comum e ocorre por meio de estolões com 30 a $60 \mathrm{~cm}$ de comprimento.

Beija-flores, borboletas e abelhas foram os visitantes das flores de $R$. brevifolia durante o período de estudo (tabela 2), visitando flores de primeiro e segundo dias. Dentre os beija-flores, Amazilia lactea foi o mais freqüente, correspondendo a cerca de $11 \%$ do total de visitas, sendo A. versicolor e Eupetomena macroura visitantes esporádicos. Phaethornis pretrei Lesson \& De Lattre e Thalurania glaucopis Gmelin (fêmea) foram visitantes raros (Ivan Sazima com. pess.), que não foram registrados durante as observações regulares. Amazilia lactea visitou as flores em intervalos 
Tabela 2. Visitantes das flores de Ruellia brevifolia, sua freqüência de visitas, categoria dos visitantes e recursos florais utilizados. $\mathrm{A}=$ polinizador principal: contata órgãos sexuais e freqüência de visita $\geq 10 \% ; \mathrm{B}=$ polinizador ocasional: contata órgãos sexuais e freqüência de visita $<10 \%$; $\mathrm{C}$ = pilhador: não contata órgãos sexuais. $\mathrm{N}=$ néctar; $\mathrm{P}=$ pólen

\begin{tabular}{|c|c|c|c|}
\hline Visitantes & $\begin{array}{c}\text { Frequiência de visitas } \\
\text { Número }(\%)\end{array}$ & $\begin{array}{c}\text { Categoria } \\
\text { dos visitantes }\end{array}$ & Recursos utilizados \\
\hline \multicolumn{4}{|l|}{ AVES } \\
\hline \multicolumn{4}{|l|}{ Trochilidae } \\
\hline Amazilia lactea Lesson & $14(10,8)$ & A & $\mathrm{N}$ \\
\hline Amazilia versicolor Vieillot & $4(3,08)$ & $\mathrm{B}$ & $\mathrm{N}$ \\
\hline Eupetomena macroura Gmelin & $4(3,08)$ & B & $\mathrm{N}$ \\
\hline \multicolumn{4}{|l|}{ INSETOS } \\
\hline \multicolumn{4}{|l|}{ LEPIDOPTERA } \\
\hline \multicolumn{4}{|l|}{ Nymphalidae } \\
\hline Heliconius ethilla narcaea Godt. & $45(34,6)$ & A & $\mathrm{N} / \mathrm{P} ?$ \\
\hline Heliconius erato phyllis $\mathrm{F}$. & $11(8,46)$ & $\mathrm{B}$ & $\mathrm{N} / \mathrm{P} ?$ \\
\hline Aeria olena Weymer & $4(3,08)$ & $\mathrm{C}$ & $\mathrm{N}$ \\
\hline \multicolumn{4}{|l|}{ Papilionidae } \\
\hline Parides agavus Drury & $5(3,8)$ & B & $\mathrm{N}$ \\
\hline Parides anchises nephalion Godt. & $4(3,08)$ & B & $\mathrm{N}$ \\
\hline Parides bunichus Hbn. & $1(0,77)$ & $\mathrm{B}$ ? & $\mathrm{N} ?$ \\
\hline \multicolumn{4}{|l|}{ Pieridae } \\
\hline Phoebis argante $\mathrm{F}$. & $17(13,1)$ & A & $\mathrm{N}$ \\
\hline Phoebis philea L. & $4(3,08)$ & $\mathrm{B}$ & $\mathrm{N}$ \\
\hline Eurema arbela Geyer & $3(2,30)$ & $\mathrm{C}$ & $\mathrm{N}$ \\
\hline \multicolumn{4}{|l|}{ Hesperiidae } \\
\hline Lychnuchoides ozias Hew. & $4(3,08)$ & B & $\mathrm{N}$ \\
\hline Lycas argentea Hew. & $2(1,54)$ & B & $\mathrm{N}$ \\
\hline \multicolumn{4}{|l|}{ HYMENOPTERA } \\
\hline \multicolumn{4}{|l|}{ Anthophoridae } \\
\hline Ceratina chloris $\mathrm{F}$. & $1(0,77)$ & $\mathrm{C}$ & $\mathrm{N} / \mathrm{P}$ \\
\hline Exomalopsis (E.) auropilosa Spinola & $1(0,77)$ & $\mathrm{C}$ & $\mathrm{N}$ \\
\hline Xylocopa sp. & $3(2,30)$ & $\mathrm{C}$ & $\mathrm{N}$ \\
\hline \multicolumn{4}{|l|}{ Apidae } \\
\hline Apis mellifera $\mathrm{L}$. & $1(0,77)$ & $\mathrm{C}$ & $\mathrm{N}$ \\
\hline \multicolumn{4}{|l|}{ Halictidae } \\
\hline Augochloropsis cupreola Cockerell & $1(0,77)$ & $\mathrm{C}$ & $\mathrm{N}$ \\
\hline Augochlora sp. & $1(0,77)$ & $\mathrm{C}$ & $\mathrm{N}$ \\
\hline
\end{tabular}

irregulares, que variaram de 5 a 60 minutos. O comportamento de visita das espécies de beija-flores é semelhante entre si: pairam defronte à flor e introduzem o bico no tubo da corola para retirar o néctar, ocasião em que contatam o estigma e as anteras com a porção superior do bico e/ou fronte. Visitam diversas flores do mesmo ou de agrupamentos próximos. Os beija-flores realizaram visitas apenas quando havia muitas flores no agrupamento.

Dentre as borboletas, Heliconius ethilla narcaea foi a mais freqüente, com aproximadamente $35 \%$ das visitas (tabela 2). Esta espécie iniciava as visitas às 9:30 h, para tomar néctar ou coletar pólen. Após pousar sobre os lobos da corola, introduzia a probóscide (figura 3) e, às vezes, a fronte no tubo floral, contatando os órgãos reprodutivos com a probóscide, fronte e/ou porção inicial das asas. Após 15 a 45 s numa flor, visitava outra da mesma inflorescência ou de ramos próximos. Periodicamente interrompia as visitas, recomeçando-as após alguns minutos, no mesmo agrupamento ou em outro nas proximidades. Suas visitas foram mais freqüentes no período da manhã. Comportamento de visita similar ao de $H$. ethilla narcaea foi apresentado por Heliconius erato phyllis, com quase $9 \%$ de freqüência de visitas (tabela 2). Indivíduos destas espécies apresentaram pólen de $R$. brevifolia principalmente na 
probóscide e cabeça. Com exceção de Phoebis argante, as demais espécies de borboletas apresentaram baixa freqüência de visitas (tabela 2). Estas espécies realizavam visitas rápidas, de 3 a $6 \mathrm{~s}$ e contatavam anteras e estigma, apresentando dimensões e comportamento de visita semelhante ao das Nymphalidae. As borboletas Aeria olena e Eurema arbela, devido ao pequeno porte não contatavam os órgãos reprodutivos das flores de $R$. brevifolia, sendo pilhadoras de néctar (tabela 2).

Cinco espécies de abelhas entravam na flor, pilhavam néctar e não contatavam o estigma; uma espécie também pilhava pólen (tabela 2). Xylocopa sp. pousava externamente sobre a corola, perfurando-a na base para retirar néctar.

\section{Discussão}

O padrão fenológico apresentado pelas populações de Ruellia brevifolia é do tipo contínuo de Newstrom et al. (1994), apesar dos indivíduos desta espécie apresentarem padrão subanual, pois nos períodos entre os picos de floração há pouca ou nenhuma flor. A floração contínua de $R$. brevifolia é mantida pela alternância de flores $\mathrm{CH}$ e $\mathrm{CL}$, portanto havendo formação de sementes durante praticamente todo o ano, o que é vantajoso para esta espécie que ocupa áreas relativamente abertas e sujeitas a diversos tipos de alterações, incluindo impacto antrópico (Pedrotti \& Guarin Neto 1998).

A alternância de ciclos casmogâmicos e cleistogâmicos apresentada por $R$. brevifolia é um fenômeno relacionado a condições ecológicas e climáticas (Sell 1977), sendo comum em espécies de Ruellia (Long 1971, 1977). A ocorrência de flores CL de $R$. brevifolia predominantemente durante os meses com baixa precipitação e temperatura, sugere que seus ciclos cleistogâmicos sejam influenciados por estes fatores ambientais, à semelhança do observado por Piovano et al. (1995) em plantas cultivadas de $R$. brevifolia e por Long (1977) e Sell (1977) para várias espécies de Ruellia. O tipo de cleistogamia apresentado por $R$. brevifolia é denominado de verdadeira ("true cleistogamy") de acordo com a definição dada por Lord (1981), uma vez que nesta espécie também ocorrem flores $\mathrm{CH}$, e as flores $\mathrm{CL}$ apresentam corola e estames reduzidos, mas gineceu e cálice com pouca ou nenhuma redução. O estigma em gancho na flor CL de $R$. brevifolia parece ser característica comum no gênero, sendo encontrado também por Long (1977) em diversas espécies de Ruellia.
Apesar das variações no comprimento do estilete, as flores $\mathrm{CH}$ de $R$. brevifolia não são heterostílicas pois não há diferenças no comprimento dos filetes. Essas variações no estilete também foram verificadas por Piovano et al. (1995) em plantas cultivadas desta espécie, cujas flores apresentaram estiletes mais longos que os estames (tipo 1, "longistilas") ou similares no comprimento (tipo 2, "brevistilas"), porém não mais curtos e nem na mesma planta, diferindo do observado neste estudo. Piovano et al. (1995) sugerem que a variação no comprimento do estilete pode estar sob controle genético pois as diferenças persistem sob condições ambientais similares, bem como os descendentes herdam o tipo de flor do parental após autopolinização.

A razão $\mathrm{P} / \mathrm{O}$ das flores CL é cerca de $60 \%$ menor que a das flores $\mathrm{CH}$, sendo em ambos os tipos florais menor que a observada por Piovano et al. (1995), o que pode estar relacionado a variações locais deste atributo nas populações, como ocorre para outras características como o tamanho da corola e a pilosidade das folhas (Ezcurra 1993). Uma vez que o número de óvulos é similar nos dois tipos florais, o decréscimo na razão $\mathrm{P} / \mathrm{O}$ é devido à redução na quantidade de grãos de pólen, fator freqüente segundo Cruden (1977). A razão $\mathrm{P} / \mathrm{O}$ das flores $\mathrm{CH}$ de $R$. brevifolia indica que é uma espécie autógama facultativa ao compararmos com os valores de razão P/O definidos por Cruden (1977). De acordo com o ISI $(1,06)$ esta espécie é autocompatível (Weller \& Ornduff 1977, Sobrevila \& Arroyo 1982). O baixo IAS $(0,32)$ provavelmente é devido ao fato da maioria $(71 \%)$ das flores $\mathrm{CH}$ apresentarem hercogamia e que correspondem, em parte, às flores do tipo 1 de Piovano et al. (1995). O percentual de frutos formados via autopolinização espontânea (30\%) é similar ao percentual de flores não hercogâmicas (29\%), indicando que nestas flores a autopolinização pode ocorrer, à semelhança do verificado nas flores tipo 2 de Piovano et al. (1995). A polinização cruzada em $R$. brevifolia é favorecida pela hercogamia e pela protoginia, aspectos que também ocorrem em doze espécies autocompatíveis de Ruellia (Long 1966). De acordo com Webb \& Lloyd (1986), a hercogamia é interpretada como uma estratégia reprodutiva que promove polinização cruzada pois evita a autopolinização espontânea. A eficácia reprodutiva $(0,62$, cf. Sobrevila \& Arroyo 1982) de $R$. brevifolia está relacionada à atividade dos polinizadores e ao fato de apresentar autopolinização espontânea. A autopolinização das flores CL e, conseqüentemente, a formação de frutos em condições naturais nestas flores é favorecida pela forma 
e posicionamento do estigma junto às anteras. A menor taxa de frutificação das flores $\mathrm{CL}$ em relação às flores $\mathrm{CH}$ pode ser, parcialmente, decorrente do fato das flores CL ocorrerem principalmente na estação seca, período do ano com condições menos favoráveis ao crescimento e desenvolvimento (Larcher 2000). A diminuição da viabilidade polínica das flores $\mathrm{CL}$ em relação às $\mathrm{CH}$, conforme também observado por Piovano et al. (1995), provavelmente também está relacionada a fatores ambientais (Shivanna \& Johri 1985).

A baixa produção de néctar em $R$. brevifolia assemelha-se à das flores de Ruellia asperula (Machado \& Sazima 1995), e a de espécies de Sinningia (Gesneriaceae) (Sazima et al. 1996) e de Esterhazya macrodonta (Scrophulariaceae) (Freitas \& Sazima 2001), contrastando com a alta produção de néctar em numerosas espécies ornitófilas (Sazima et al. 1996, Buzato et al. 2000). A baixa quantidade de néctar é atributo que pode ser importante na seleção dos polinizadores, uma vez que os beija-flores foram avistados apenas em agrupamentos de plantas em pico de floração, ou seja, quando há grande número de flores, tornando alta a quantidade de néctar na população. Além disso, os beija-flores visitaram preferencialmente durante o período da manhã, ocasião em que, de acordo com Piovano et al. (1995), ocorre pico de secreção de néctar nesta espécie. Ainda, segundo Piovano et al. (1995), a taxa de secreção de néctar nas flores de $R$. brevifolia declina com sua remoção. Sendo assim, a baixa quantidade de néctar produzida em agrupamentos com poucas flores e seu declínio após a remoção poderia desestimular visitas subseqüentes de beija-flores como ocorre em E. macrodonta (Scrophulariaceae, Freitas \& Sazima 2001).

A concentração de açúcares no néctar de $R$. brevifolia é semelhante à de $R$. asperula (Machado \& Sazima 1995) e é considerada típica para diversas espécies polinizadas por beija-flores (Baker 1975, Passos \& Sazima 1995, Piovano et al. 1995, Sazima et al. 1996, Buzato et al. 2000).

As flores $\mathrm{CH}$ de $\mathrm{R}$. brevifolia apresentam atributos associados às síndromes de ornitofilia e psicofilia definidos por Faegri \& van der Pijl (1979) como, corola vistosa, tubulosa e grande distância entre anteras e estigma e a fonte de alimento. Entretanto, outros caracteres como ausência de odor e de plataforma de pouso, bem como a posição ligeiramente pendente destas flores, são relacionadas principalmente à sindrome de ornitofilia.

Dentre os visitantes, o beija-flor Amazilia lactea e as borboletas Heliconius ethilla narcaea e Phoebis argante são os polinizadores principais de $R$. brevifolia, devido ao comportamento e freqüência de visitas, totalizando juntos ca. 58\% do total de visitas. Polinização por beija-flores e borboletas foi também registrada em espécies de Ipomoea (Convolvulaceae) invasoras de culturas (Machado \& Sazima 1987). Embora menos frequiente que as borboletas a participação de A. lactea é importante na polinização de $R$. brevifolia, pois visita grande número de flores em curto período de tempo; além disso, sua estratégia de forrageamento é do tipo linha-de-captura, resultando em maior fluxo e dispersão de pólen do que o realizado por borboletas em geral (Webb \& Bawa 1983). As demais espécies de beijaflores são consideradas polinizadoras ocasionais e apresentam estratégia de forrageamento semelhante a de A. lactea. A baixa quantidade de recursos em $R$. brevifolia provavelmente promove esta estratégia de forrageamento, que por sua vez favorece a polinização cruzada (Frankie \& Haber 1983, Endress 1994). As outras espécies de borboletas são consideradas polinizadoras ocasionais de $R$. brevifolia, com exceção de Aeria olena e Eurema arbela, consideradas pilhadoras, bem como as abelhas que apresentam um tipo de pilhagem semelhante ao que ocorre em $R$. asperula (Machado \& Sazima 1995).

Em Ruellia brevifolia o florescimento contínuo, a cleistogamia sazonal, a autocompatibilidade, a reprodução vegetativa, bem como a utilização de dois grupos de polinizadores são características que favorecem a ocupação e a permanência desta espécie em habitats sujeitos a alterações.

Agradecimentos - A Ivan Sazima por observações de campo e identificação dos beija-flores, Keith S. Brown Jr. pela identificação das borboletas, João M. F. Camargo pela identificação das abelhas, Josué Raizer pela confecção do gráfico, Erich A. Fischer pela análise estatística, José I. Cunha pelo auxílio no trabalho de campo, Altino A. Ortolani, do Instituto Agronômico de Campinas, pelo fornecimento dos dados climáticos e Douglas de A. Benitez, pela editoração da figura 3. CNPq.

\section{Referências bibliográficas}

ALEXANDER, M.P. 1980. A versatile stain for pollen, fungi, yeast and bacteria. Stain Technology 55:13-18.

BAKER, H.G. 1975. Sugar concentrations in nectars from hummingbird flowers. Biotropica 7:37-41.

BUZATO, S., SAZIMA, M. \& SAZIMA, I. 2000. Hummingbirdpollinated floras at three Atlantic Forest sites. Biotropica 32:824-841.

CRUDEN, R.W. 1977. Pollen-ovules ratios: a conservation indicator of breeding systems in flowering plants. Evolution 31:32-46. 
DAFNI, A. 1992. Pollination ecology: a practical approach. Oxford University Press, New York.

ENDRESS, P.K. 1994. Diversity and evolutionary biology of tropical flowers. Cambridge University Press, Cambridge.

EZCURRA, C. 1989. Ruellia sanguinea (Acanthaceae) y especies relacionadas en Argentina, Uruguay y sur de Brasil. Darwiniana 29:269-287.

EZCURRA, C. 1993. Systematics of Ruellia (Acanthaceae) in southern South America. Annals of the Missouri Botanical Garden 80:787-845.

EZCURRA, C. \& AZKUE, D. 1989. Validation and genetic and morphological relationships of Ruellia macrosolen (Acanthaceae) from southern South America. Systematic Botany 14:297-303.

FAEGRI, K. \& Van der PIJL, L. 1979. The principles of pollination ecology. Pergamon Press, Oxford.

FRANKIE, G.W. \& HABER, W.A.1983. Why bees move among mass-flowering neotropical trees. In Handbook of pollination biology (C.E. Jones \& R.J. Little, eds.). Van Nostrand Reinhold Company Inc., New York, p.360-372.

FREITAS, L. \& SAZIMA, M. 2001. Nectar features in Esterhazya macrodonta, a hummingbird-pollinated Scrophulariaceae in southeastern Brazil. Journal of Plant Research 114:187-191.

JOHANSEN, D.A. 1940. Plant microtechnique. McGraw-Hill Book Company, New York.

KNUTH, P. 1905. Handbuch der Blütenbiologie. Engelmann, Leipzig.

LARCHER, W. 2000. Ecofisiologia vegetal. Rima Artes e Textos, São Carlos.

LONG, R.W. 1966. Artificial interespecific hybridization in Ruellia (Acanthaceae). American Journal of Botany 53:917-927.

LONG, R.W. 1971. Floral polymorphy and amphimitic breeding systems in Ruellia carolinensis (Acanthaceae). American Journal of Botany 58:525-531.

LONG, R.W. 1977. Artificial induction of obligate cleistogamy in species-hybrids in Ruellia (Acanthaceae). Bulletin of the Torrey Botanical Club 104:53-56.

LORD, E.M. 1981. Cleistogamy: a tool for the study of floral morphogenesis, function and evolution. Botanical Review 47:421-449.

MACHADO, I.C.S. \& SAZIMA, M. 1987. Estudo comparativo da biologia floral em duas espécies invasoras: Ipomoea hederifolia e I. quamoclit (Convolvulaceae). Revista Brasileira de Biologia 47:425-436.

MACHADO, I.C. \& SAZIMA, M. 1995. Biologia da polinização e pilhagem por beija-flores em Ruellia asperula Lindau (Acanthaceae) na caatinga, nordeste brasileiro. Revista Brasileira de Botânica 18:27-33.

MORELLATO, P.C. \& LEITÃOFILHO, H.F. 1996. Reproductive phenology of climbers in a southeastern Brazilian forest. Biotropica 28:180-191.
NEWSTROM, L.E., FRANKIE, G.W. \& BAKER, H.G. 1994. A new classification for plant phenology based on flowering patterns in lowland tropical forest trees at $\mathrm{La}$ Selva, Costa Rica. Biotropica 26:141-159.

PASSOS, L. \& SAZIMA, M. 1995. Reproductive biology of distylous Manettia luteo-rubra (Rubiaceae). Botanica Acta 108:309-313.

PEDROTTI, D.E. \& GUARIN NETO, G. 1998. Flora ruderal da cidade de Cuiabá, Mato Grosso, Brasil. Acta Botanica Brasilica 12:135-143.

PIOVANO, M., GALETTO, L. \& BERNARDELLO, L. 1995. Floral morphology, nectar features and breeding system in Ruellia brevifolia (Acanthaceae). Revista Brasileira de Biologia 55:409-418.

RADFORD, A.E., DICKINSON, W.C., MASSEY, J.R. \& BELL, C.R. 1974. Vascular plant systematics. Harper \& Row Publishers, New York.

RAGHUVANSHI, S.S., PATHAK, C.S. \& SINGH, R.R. 1981. Gibberellic acid response and induced chasmogamous variant in cleistogamous Ruellia hibrid (R. tweediana $\mathrm{x}$ R. tuberosa). Botanical Gazette 142:40-42.

RAMAMOORTHY, T.P. 1988. A new species of Ruellia (Acanthaceae) from western Mexico. Annals of the Missouri Botanical Garden 75:1664-1665.

SAZIMA, I., BUZATO, S. \& SAZIMA, M. 1996. An assemblage of hummingbird-pollinated flowers in a montane forest in southeastern Brazil. Botanica Acta 109:149-160.

SELL, Y. 1977. La cléistogamie chez Ruellia lorentziana Griseb. et quelques autres Acanthacées. Berichte der Deutschen Botanischen Gesellschaft 90:135-147.

SHIVANNA, K.R. \& JOHRI, B.M. 1985. The angiosperm pollen: structure and function. John Wiley \& Sons, New York.

SOBREVILA, C. \& ARROYO, M.T.K. 1982. Breeding systems in a montane tropical cloud forest in Venezuela. Plant Systematics and Evolution 140:19-37.

SOKAL, R.R. \& ROHLF, F.J. 1979. Biometría. H. Blume Ediciones, Madrid.

WEBB, C.J. \& BAWA, K.S. 1983. Pollen dispersal by hummingbird and butterflies: a comparative study in two lowland tropical plants. Evolution 37:1258-1270.

WEBB, C.J. \& LLOYD, D.G. 1986. The avoidance of interference between the presentation of pollen and stigmas in angiosperms. II. Herkogamy. New Zealand Journal of Botany 24:163-178.

WELLER, S.G. \& ORNDUFF, R. 1977. Cryptic selfincompatibility in Amsinckia grandiflora. Evolution 31:47-51.

WITZTUM, A. \& SCHULGASSER, K. 1995. The mechanics of seed expulsion in Acanthaceae. Journal of Theoretical Biology 176:531-542. 